\title{
Glial Cell Line-Derived Neurotrophic Factor Increases Stimulus-Evoked Dopamine Release and Motor Speed in Aged Rhesus Monkeys
}

\author{
Richard Grondin, ${ }^{\star}$ Wayne A. Cass, ${ }^{\star}$ Zhiming Zhang, John A. Stanford, Don M. Gash, and Greg A. Gerhardt \\ Department of Anatomy and Neurobiology and Morris K. Udall Parkinson's Disease Research Center of Excellence, University of Kentucky Medical Center, \\ Lexington, Kentucky 40536-0298
}

Changes in the functional dynamics of dopamine release and regulation in the basal ganglia have been posited to contribute to age-related slowing of motor functions. Here, we report the effects of glial cell line-derived neurotrophic factor (GDNF) on the stimulus-evoked release of dopamine and motor speed in aged monkeys (21-27 years of age; $n=10)$. Although no changes were observed in the vehicle controls $(n=5)$, chronic infusions of $7.5 \mu \mathrm{g}$ of GDNF per day for 2 months into the right lateral ventricle initially increased hand movement speed up to $40 \%$ on an automated hand-reach task. These effects were maintained for at least 2 months after replacing GDNF with vehicle, and increased up to another $10 \%$ after the reinstatement of GDNF treatment for 1 month. In addition, upper-limb motor performance times of the aged GDNF-treated animals $(n=5)$ recorded at the end of the study were similar to those of five young adult monkeys (8-12 years of age). The stimulus-evoked release of dopamine was significantly increased, up to $130 \%$ in the right caudate nucleus and putamen and up to $116 \%$ in both the right and left substantia nigra of the aged GDNF recipients compared with vehicle controls. Also, basal extracellular levels of dopamine were bilaterally increased, up to $163 \%$ in the substantia nigra of the aged GDNFtreated animals. The data suggest that the effects of GDNF on the release of dopamine in the basal ganglia may be responsible for the improvements in motor functions and support the hypothesis that functional changes in dopamine release may contribute to motor dysfunctions characterizing senescence.

Key words: chronic GDNF; aging; rhesus monkeys; in vivo microdialysis; basal ganglia; motor speed

\section{Introduction}

The progressive slowing of motor functions and the development of parkinsonian signs are common features seen with advancing age in humans (Bennett et al., 1996; Smith et al., 1999). As in elderly humans, motor functions in monkeys also decline with age, as seen by decreased home cage activity and increased reaction times compared with young adult monkeys (Bachevalier et al., 1991; Irwin et al., 1994; Ovadia et al., 1995; Emborg et al., 1998; Zhang et al., 2000). In correspondence with human aging, aged monkeys are also significantly slower on tasks involving fine motor hand movements than are young adult monkeys, and often display two or more parkinsonian signs (Emborg et al., 1998; Gash et al., 1999; Zhang et al., 2000).

Several alterations of the nigrostriatal dopaminergic system are seen with aging in humans and monkeys. These age-related changes include decreased tissue levels of nigrostriatal dopamine, decreased levels of tyrosine hydroxylase and dopamine trans-

Received June 13, 2002; revised Dec. 18, 2002; accepted Dec. 18, 2002.

This work was supported by a postdoctoral fellowship award from the Fond de la Recherche en Santé du Québec (R.G.) and by United States Public Health Service Grants NS39787, AG13494, AG06434, and MH01245. We thank Dr. Michael Klein at Amgen Inc. for providing GDNF, Dr. Dennis Elseberry at Medtronic Inc. for providing the pumps and associated hardware and software, and Robin Avison, Tracy Barber, Michael Harned, Li-Ya Liu, Aaron Loveland, Sheila McLean, Laura Peters, and Ashley Walton for excellent technical support.

${ }^{*}$ R.G. and W.A.C. contributed equally to this work.

Correspondence should be addressed to Dr. Richard Grondin, Anatomy and Neurobiology, University of Kentucky Medical Center, 305 Davis Mills Building, Magnetic Resonance Imaging and Spectroscopy Center, Lexington, KY 40536-0098. E-mail: regrom0@pop.uky.edu

Copyright $\odot 2003$ Society for Neuroscience $\quad 0270-6474 / 03 / 231974-07 \$ 15.00 / 0$ porter, and decreased stimulus-evoked release of nigrostriatal dopamine (Bannon et al., 1992; Kish et al., 1992; Irwin et al., 1994; Gerhardt et al., 1995, 2002; Emborg et al., 1998). As in Parkinson's disease, these data suggest that the nigrostriatal dopaminergic system may contribute to age-associated motor dysfunctions seen in humans and monkeys. However, unlike Parkinson's disease, the extensive loss $(>60 \%)$ of dopamine neurons in the pars compacta of the substantia nigra does not appear to occur in normal aging, either in human senescence or in animal models of aging (Fearnley and Lees, 1991; Irwin et al., 1994; Emborg et al., 1998; Kubis et al., 2000; Gerhardt et al., 2002). Therefore, midbrain dopaminergic cell loss alone cannot explain ageassociated motor deficits. Rather, changes in the functional dynamics of dopamine release and regulation in the striatum and/or the substantia nigra might better explain the motor declines seen during aging in nonhuman primates, and possibly in humans (Gerhardt et al., 2002). If so, interventions that would upregulate dopamine release in the basal ganglia should improve the motor deficits characterizing normal aging.

The potent dopaminergic trophic factor glial cell line-derived neurotrophic factor (GDNF) offers a promising approach to potentially repair and restore function to damaged dopaminergic neurons (Gash et al., 1998). It has been hypothesized that GDNF mediates its effects, at least in part, by regulating the neuronal excitability of midbrain dopaminergic neurons (Yang et al., 2001). This GDNF-induced potentiation of neuronal excitability would result in an increase in dopamine release, leading to an enhancement in motor functions (Yang et al., 2001). A previous 
report by Kordower et al. (2000) investigated the effects of GDNF in aged monkeys. In this study, viral vector delivery of GDNF into the nigrostriatal dopaminergic system (striatum and substantia nigra) of four aged monkeys was shown to increase tissue levels of dopamine in the striatum and to increase the number of tyrosine hydroxylase-immunoreactive (TH-IR) nigral neurons. However, the effects of GDNF on motor behavior and dopamine release in aged monkeys were not described and remain unclear. Thus, the present study was designed to determine whether the intraventricular infusion of exogenous GDNF can improve motor deficits in aged monkeys, and if so, whether this improvement is associated with an increase in nigrostriatal dopamine release. The 10 aged rhesus monkeys used in this study ranged in age from 21 to 27 years, approximately equivalent to 63-81 years of age in humans (Andersen et al., 1999). For comparison, a group of five young adult rhesus monkeys ( $8-12$ years of age) was tested in parallel on the same hand-reach motor task.

\section{Materials and Methods}

Animals. All procedures were conducted in the Laboratory Animal Facilities of the University of Kentucky, which are fully accredited by the Association for Assessment and Accreditation of Laboratory Animal Care. The Animal Care and Use Committee of the University of Kentucky approved all protocols. In addition, veterinarians skilled in the health care and maintenance of nonhuman primates supervised all animal care.

Five young adult (mean age, $10.3 \pm 1.2$ years) and 10 aged (mean age, $23.8 \pm 0.6$ years) female rhesus monkeys (Macaca mulatta) were obtained from a commercial supplier (Covance, Alice, TX) and used in this study. The 10 aged rhesus monkeys were matched for age and were randomly divided on arrival into two groups of five animals. The animals were housed in temperature-controlled rooms and maintained on a $12 \mathrm{hr}$ light/dark cycle. Their diet consisted of certified primate biscuits supplemented daily with fresh fruit or vegetables; water was available ad libitum. All animals were weighed weekly during the course of the study.

Surgical procedures. Under sterile field conditions, a catheter $(1 \mathrm{~mm}$ outer diameter, model 8770AS; Medtronic, Minneapolis, MN) was stereotactically implanted adjacent to the striatum into the right lateral ventricle of the 10 aged animals. The brain coordinates for intraventricular implantation were determined by magnetic resonance imaging (MRI) before the surgery. The ventricular catheter has a hole in the tip, with two adjacent side holes for drug delivery. The catheter was subcutaneously connected via flexible polyurethane tubing to a SynchroMed programmable pump (model 8616-10; Medtronic), that was implanted subcutaneously in the lateral abdominal region as described by Grondin et al. (2001). The animals were anesthetized with isoflurane (1-3\%) during these procedures and were allowed 2 weeks to recover from the surgery. Placement of the catheter into the right lateral ventricle was verified by MRI. The right lateral ventricle was chosen to parallel our studies conducted in hemiparkinsonian monkeys (Gash et al., 1996), whereas programmable pumps were used to parallel the prolonged delivery of GDNF studied previously in aged monkeys using viral vectors (Kordower et al., 2000).

Drug treatments. Vehicle (10 mm citrate, $150 \mathrm{~mm} \mathrm{NaCl}$ buffer; Amgen Inc., Thousand Oaks, CA) was infused throughout the 6 month study in five control animals ( $23.5 \pm 1$ years of age). The other five aged animals $(24.1 \pm 0.7$ years of age $)$ were initially administered recombinant methionine human GDNF (Amgen) for 3 months. Because a pilot experiment had shown that the threshold for inducing behavioral improvements in monkeys was $>3.75 \mu \mathrm{g} / \mathrm{d}$ GDNF (Grondin et al., 1998), the aged GDNF recipients received a daily dose of $7.5 \mu \mathrm{g}$ of GDNF that was delivered over $24 \mathrm{hr}$ in a volume of $100 \mu \mathrm{l}$ of citrate buffer (a $13 \mu \mathrm{l}$ bolus of $0.075 \mathrm{mg} / \mathrm{ml}$ GDNF delivered every $6 \mathrm{hr}$ in addition to a basal flow rate of $2 \mu \mathrm{l} / \mathrm{hr}$ between each bolus). Vehicle-treated animals received the same daily volume of citrate buffer. The pumps were refilled every 4 weeks with either GDNF or vehicle by injections through the skin into a fill port (Grondin et al., 2001, 2002a,b).
Behavioral testing was interrupted during the third month of chronic GDNF infusion, during which microdialysis experiments were conducted (weeks 9-12). Behavioral evaluations were resumed on weeks 13-20, during which all 10 aged animals received vehicle (washout period). This washout period with the vehicle was conducted to determine whether the behavioral effects would persist in the absence of GDNF. Finally, reinstatement of treatment for 1 month was also performed to determine whether GDNF could sustain or additionally increase motor speed after a washout period. The study was terminated 6 months after beginning treatment with either vehicle or GDNF (week 24).

Monkey movement analysis panel. We used our previously described automated monkey movement analysis panel (mMAP) to objectively quantify the upper-limb motor performance time of the animals retrieving a miniature marshmallow from a platform placed in a receptacle chamber attached to the home cage (Gash et al., 1999; Grondin et al., 2000). The aged animals were first trained to a plateau level of performance before receiving GDNF or vehicle. Data recorded during the last of four testing sessions before initiating drug treatment were used as a baseline. Testing occurred weekly and consisted of 12 trials alternating between the right (six trials) and left (six trials) hand using the appropriate armhole portal giving access to the chamber. The data were analyzed using two parameters: fine motor performance time and coarse motor performance time. On each trial, fine motor performance time was considered to be the time the hand was in the receptacle picking up the food reward. Coarse motor performance time was recorded as the time for the upper limb to move from the armhole portal to the food receptacle portal and then back from the receptacle portal into the cage (i.e., the total time for a trial minus the fine motor performance time). In addition, a group of five young adult monkeys ( $10.3 \pm 1.2$ years of age) was preadapted to routinely retrieve food from the receptacle chamber with each hand. Baseline data were then collected using the platform task level, in parallel with the aged animals. The peak performance recorded for each young adult monkey was used for comparison.

In vivo microdialysis. The brain coordinates for microdialysis probe implantation were determined by MRI before the surgery (Gerhardt et al., 2002). Nine weeks after beginning either vehicle or GDNF administration, the aged animals were anesthetized with isoflurane (1-3\%) and placed in an MRI-compatible Kopf stereotaxic apparatus (David Kopf Instruments, Tujunga, CA). Under sterile field conditions, small holes were drilled in the skull over the left and right substantia nigra. Custommade CMA/11 dialysis probes with a membrane length of $3 \mathrm{~mm}$ and diameter of $0.24 \mathrm{~mm}$ (CMA Microdialysis, North Chelmsford, MA) were then positioned bilaterally in the substantia nigra and perfused continuously at a rate of $1.2 \mu \mathrm{l} / \mathrm{min}$ with artificial CSF (in mM: $145 \mathrm{NaCl}, 2.7 \mathrm{KCl}$, $1.2 \mathrm{CaCl}_{2}, 1.0 \mathrm{MgCl}_{2}, 0.2$ ascorbic acid, and $2.0 \mathrm{NaH}_{2} \mathrm{PO}_{4}, \mathrm{pH} 7.4$ ). Microdialysate fractions were collected at $30 \mathrm{~min}$ intervals. After a $1 \mathrm{hr}$ application of artificial CSF to collect baseline fractions, excess potassium $(100 \mathrm{~mm} \mathrm{KCl}, 47.7 \mathrm{~mm} \mathrm{NaCl})$ was then included in the perfusate for a single $30 \mathrm{~min}$ fraction $\left(t_{0}-t_{30}\right)$. Two hours later, $250 \mu \mathrm{M}$ amphetamine was included in the perfusate for a single $30 \mathrm{~min}$ fraction $\left(t_{120}-t_{150}\right)$. Three additional fractions were collected after discontinuing amphetamine administration $\left(t_{180}-t_{240}\right)$. Two weeks later (week 11), the same procedures were repeated ipsilateral to the infusion site, in the right caudate nucleus and the right putamen. Microdialysate fractions were analyzed using standard HPLC procedures coupled with electrochemical detection (Cass, 1996; Gerhardt et al., 2002). Microdialysis data were expressed as the concentration of dopamine, homovanillic acid (HVA), or 3,4-dihydroxyphenylacetic acid (DOPAC) in the dialysates.

Statistical analysis. The mMAP data represent an average of the left and right upper-limb motor time values, because there were no significant differences observed between the left and right limb values. Upper-limb motor performance times recorded in the aged animals were analyzed using repeated-measures ANOVA, with treatment as the betweensubjects factor and week of treatment as the within-subjects factor, followed by Newman-Keuls post hoc comparisons. The peak performance times obtained in the young adults and the aged GDNF-treated recipients (week 24) were compared using two-tailed independent-samples $t$ tests.

ANOVA, with treatment as the between-subjects factor and the side of brain as the within-subjects factor, was used to estimate differences in 
nigral basal levels of dopamine, DOPAC, or HVA between aged animals in the control and GDNF treatment groups, followed by Newman-Keuls post hoc comparisons. Basal levels of extracellular dopamine, DOPAC, or HVA in the caudate and putamen were analyzed separately using independent-samples $t$ tests. Basal levels were defined as the average value of the two fractions preceding stimulation by excess potassium. For statistical analysis of the stimulus-evoked overflow of dopamine, the data were separated into potassium-evoked overflow (30-120 min) and amphetamineinduced overflow (150-240 $\mathrm{min})$. The data were then analyzed using three- or two-factor repeated-measures ANOVA, with treatment as the between-subjects factor and time of collection and side of the brain (nigra only) as the within-subjects factor, followed by NewmanKeuls post hoc comparisons (Cass and Manning, 1999). $p \leq 0.05$ was considered significant in all analyses.

\section{Results}

\section{General observations}

All 10 aged animals recovered without incident after placement of the catheter into the lateral ventricle or after in vivo microdialysis procedures in the striatum and substantia nigra. A repeated-measures ANOVA, with treatment as the betweensubjects factor and week of treatment as the within-subjects factor, revealed no significant effects of chronic GDNF treatment on body weight compared with vehicle treatment ( $p=0.551$; data not shown). No other adverse effects (e.g., vomiting, self-mutilation, or dyskinesias) were observed during the course of the 6 month study.

\section{Motor function}

The mMAP data represent an average of the left and right upper-limb motor time values, because there were no significant differences observed between the left- and right-limb values. As quantified using the mMAP, the fine (hand) and coarse (arm) motor performance times of the GDNF recipients were not significantly different from those of the vehicle recipients before initiating chronic GDNF treatment (Fig. $1 A, B$ ). The fine motor performance times of the vehicle-treated animals did not improve throughout the study, whereas their coarse motor performance times were improved only at weeks 4 and 24 compared with their baseline performance before the vehicle infusion (Fig. $1 A, B)$. In contrast, the fine motor performance times of the GDNF recipients were significantly improved compared with their baseline performance before infusing GDNF or compared with the vehicle recipients (Fig. $1 A$ ). A steady improvement (up to $40 \%$ ) was initially observed in the GDNF-treated animals during the first 2 months after GDNF infusion (Fig. $1 A$ ). These effects were retained during the 2 month washout period with the vehicle and increased additionally up to $50 \%$ after the reinstatement of GDNF treatment. Also, a significant and sustained improvement in coarse motor performance time was seen during the study in the GDNF recipients compared with the vehicle recipients, starting at week 6 (Fig. $1 B$ ).

Compared with the fine motor movement time of the aged animals before receiving GDNF $(0.535 \pm 0.05 \mathrm{sec})$, the young monkeys were twice as fast at retrieving the food item from the receptacle chamber (i.e., $0.250 \pm 0.013 \mathrm{sec}$ ) (Fig. 2 A). However, the fine motor performance time of the aged GDNF-treated animals $(0.282 \pm 0.012 \mathrm{sec})$ recorded at the end of the study was comparable with that of the young adult monkeys, tested in parallel on the same automated hand-reach task (Fig. $2 \mathrm{~A}$ ). Also, as seen in Figure 2, the coarse motor performance time of the aged animals before receiving GDNF $(0.458 \pm 0.024 \mathrm{sec})$ did not significantly differ from the coarse motor performance time of the young animals (i.e., $0.418 \pm 0.027 \mathrm{sec}$ ) (Fig. 2 B). In contrast, the coarse motor performance time of the aged GDNF-treated animals observed by the end of the study was better than that of the young adult monkeys. Indeed, the average coarse motor time of 

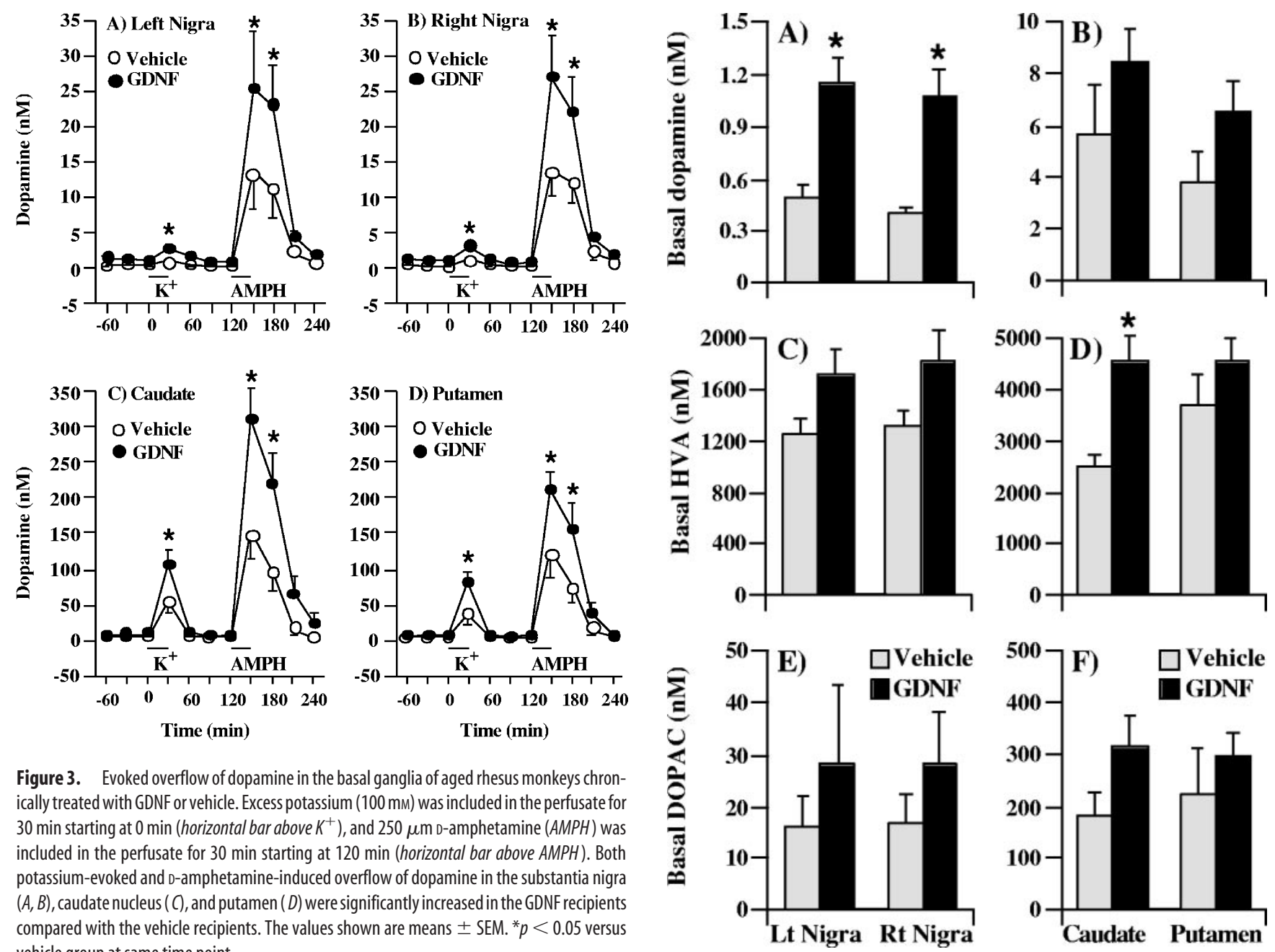

Figure 3. Evoked overflow of dopamine in the basal ganglia of aged rhesus monkeys chronically treated with GDNF or vehicle. Excess potassium ( $100 \mathrm{~mm}$ ) was included in the perfusate for 30 min starting at $0 \mathrm{~min}$ (horizontal bar above $\mathrm{K}^{+}$), and $250 \mu \mathrm{m}$ D-amphetamine (AMPH) was included in the perfusate for 30 min starting at 120 min (horizontal bar above AMPH). Both potassium-evoked and D-amphetamine-induced overflow of dopamine in the substantia nigra $(A, B)$, caudate nucleus $(C)$, and putamen $(D)$ were significantly increased in the GDNF recipients compared with the vehicle recipients. The values shown are means \pm SEM. ${ }^{*} p<0.05$ versus vehicle group at same time point.

the aged GDNF-treated animals $(0.310 \pm 0.019 \mathrm{sec})$ was comparable with that of the fastest of the young animals (i.e., $0.320 \mathrm{sec}$ ).

\section{Microdialysis}

In vivo microdialysis was used to investigate the dynamics of dopamine release in the basal ganglia of aged rhesus monkeys. Values were not corrected for in vitro recoveries, because uncorrected values may be better correlated to true values (Glick et al., 1994). Measurements were performed bilaterally in the substantia nigra and unilaterally in the right striatum (caudate nucleus and putamen) at 9 and 11 weeks, respectively, after beginning chronic infusions of either vehicle or GDNF into the right lateral ventricle. Chronic treatment with GDNF led to a significant augmentation of dopamine overflow in the basal ganglia, namely, the substantia nigra, caudate nucleus, and putamen (Fig. $3 A-D$ ). In the substantia nigra of the GDNF recipients, potassium-evoked overflow of dopamine was significantly increased, by $116 \%$ on the left side and by $110 \%$ on the right side, compared with vehicle recipients (Fig. 3A,B). Similarly, the amphetamine-induced overflow of dopamine was significantly increased, by $86 \%$ in the left substantia nigra and by $95 \%$ in the right substantia nigra, compared with vehicle recipients (Fig. $3 A, B$ ). No significant differences were seen between the left and right sides of the substantia nigra. The potassium-evoked overflow of dopamine was also increased, by $100 \%$ in the caudate nucleus and by $130 \%$ in the putamen of the GDNF-treated animals (Fig. 3C,D). The amphetamine-induced overflow of dopamine in the caudate nu-

Figure 4. Basal dialysate levels of dopamine and dopamine metabolites in the basal ganglia of aged rhesus monkeys chronically treated with GDNF or vehicle. For each compound, the basal level was defined as the average value of the two fractions preceding stimulation by excess potassium. Basal levels of extracellular dopamine were significantly increased in the substantia nigra of the GDNF-treated animals $(A)$, whereas HVA levels were significantly increased in the caudate nucleus $(D)$. The values shown are means \pm SEM. ${ }^{*} p<0.05$ versus vehicle group. $L t$, Left; $R t$, right.

cleus and putamen was increased by 100 and $66 \%$, respectively, in the GDNF recipients compared with vehicle recipients (Fig. 3C,D).

In addition, basal levels of extracellular dopamine were significantly increased, by $137 \%$ in the left substantia nigra and by $163 \%$ in the right substantia nigra of the GDNF-treated animals (Fig. 4A). Again, no significant differences were seen between the left and right sides of the substantia nigra. In the caudate nucleus and putamen, there was a nonsignificant trend for increased basal levels of extracellular dopamine (Fig. $4 B$ ). Only HVA levels were significantly increased, by $82 \%$ in the caudate nucleus of the GDNF-treated animals (Fig. 4D).

\section{Discussion}

\section{Motor function}

Studies conducted in aged rats have demonstrated that GDNF treatment can improve motor functions (Bowenkamp et al., 1996; Hebert and Gerhardt, 1997; Lapchak et al., 1997b). In the current study, although their coarse motor times were improved at weeks 4 and 24, the vehicle-treated animals did not improve their fine motor performance times on the motor task through- 
out the study. However, the aged rhesus monkeys administered GDNF were significantly faster on tasks involving both fine (hand) and coarse (arm) motor movements of the upper limb, compared with their baseline performance before infusing GDNF or compared with the vehicle recipients. For each animal, the data recorded during the last of four testing sessions before initiating drug treatment were used as a baseline. We have shown previously that aged rhesus monkeys reach a plateau level of performance by the third session when tested on the platform task (Zhang et al., 2000). Therefore, the improvement in motor functions seen in the GDNF recipients is likely to be GDNF-related, because the animals were first trained to a plateau level of performance with each hand before the infusion of GDNF, virtually eliminating the possibility of a learning effect. Alternatively, GDNF might be increasing motivation rather than motor skills per se, or it might be doing both. For instance, GDNF was shown to improve motor functions in rats and monkeys in experimental settings in which no rewards were given to the animals and in which motivation would not be expected to play a primary role, such as home-cage activity levels (Hebert and Gerhardt, 1997; Grondin et al., 2002b). This would suggest that GDNF, by its effects on dopamine, is most likely improving motor skills rather than increasing motivation. However, the effects of GDNF on motivation cannot be ruled out, particularly in a task in which it is hard to separate motivational from motor effects, such as in our food-retrieval task, which entails removing food items from a receptacle chamber. It remains unclear why the vehicle-treated animals showed improvement in their coarse motor times at weeks 4 and 24.

Interestingly, the fine and coarse motor performance times of the aged GDNF recipients recorded at the end of the study were comparable with or better than those of five young adult rhesus monkeys tested on the same task. Indeed, the average coarse motor time of the aged GDNF-treated animals was comparable with that of the fastest of the young animals. Accordingly, we have shown previously that the upper-limb motor performance of aged monkeys administered the indirect dopamine agonist levodopa or the selective dopamine uptake inhibitor 1-(2-(bis(4fluorophenyl)methoxy)ethyl)-4-(3-phenylpropyl) (GBR-12909) piperazine was not only improved compared with their baseline performance times, but was similar to that of young adult rhesus monkeys administered saline (Grondin et al., 2000). The observation that GDNF can significantly improve motor functions in aged rhesus monkeys to a performance level similar to that of young adults is important, considering that a major hallmark of aging in humans is the slowing of motor movements (Bennett et al., 1996; Smith et al., 1999).

This study also demonstrates that low doses of GDNF chronically infused into the lateral ventricle promote behavioral improvements that are maintained over time in aged rhesus monkeys, even in the absence of GDNF for up to 2 months. First, these data suggest that GDNF receptors are not prone to desensitization when chronically stimulated for several consecutive weeks. In accordance, we have observed sustained improvements in motor functions in 1-methyl-4-phenyl-1,2,3,6-tetrahydropyridine (MPTP)-exposed parkinsonian monkeys chronically treated with GDNF for 12 weeks (Grondin et al., 2002a). Second, the accumulation of radiolabeled GDNF in the nonhuman primate brain peaks between 48 and $72 \mathrm{hr}$ after a single intraventricular injection (Lapchak et al., 1998). Similarly, radiolabeled GDNF accumulates in the rodent brain within $24 \mathrm{hr}$ of a single intraventricular injection, but only trace amounts of GDNF were detected 1 week after the administration (Lapchak et al., 1997a). This in- dicates that intraventricular GDNF accumulates in the brain parenchyma to stimulate targeted neurons and is then rapidly degraded or cleared from the structure. However, in the present study, the effects of GDNF on motor performance persisted for several weeks after replacing GDNF with the vehicle, long after clearance from the basal ganglia. Accordingly, previous studies have reported long-lasting motor effects of GDNF of up to 1 month after a single intracerebral injection in MPTP-treated rhesus monkeys (Gash et al., 1996). Our data suggest that continuous stimulation of GDNF receptors may not be required for GDNF to improve motor functions effectively. This observation could have a significant impact on the future use of GDNF as a treatment for neurodegenerative diseases such as Parkinson's disease (Brundin, 2002). Consequently, additional studies are needed to determine whether improved motor behavior after chronic GDNF infusion can be sustained for $>2$ months in the absence of GDNF and whether the response would be different in aged versus MPTPlesioned rhesus monkeys modeling Parkinson’s disease.

\section{Dopaminergic functions}

In the present study, potassium-evoked and D-amphetamineinduced overflow of dopamine were significantly increased (more than twofold) in the caudate nucleus, in the putamen, and in both the left and right substantia nigra of the GDNF recipients compared with vehicle recipients, $9-11$ weeks after beginning the chronic infusion of either GDNF or vehicle. In comparison, the amphetamine-induced release of dopamine observed in the putamen $(222 \pm 26 \mathrm{nM})$ and in the substantia nigra $(27 \pm 6 \mathrm{nM}$, right side; $26 \pm 8$, left side) of the GDNF-treated aged monkeys was similar to the amphetamine-induced response seen previously in the putamen $(185 \pm 31 \mathrm{nM})$ and in the substantia nigra $(23 \pm 2$ nM) of young adult rhesus monkeys (Gerhardt et al., 2002). This suggests not only that the chronic intraventricular administration of GDNF in aged rhesus monkeys upregulates dopaminergic release processes in nigrostriatal dopamine neurons, but that GDNF can augment the functional capacity of dopamine neurons in aged monkeys to the same degree as seen in young adult rhesus monkeys. These observations also indicate that GDNF receptors and coupling mechanism(s) remain functional during aging. The drug D-amphetamine releases dopamine in a calciumindependent but transporter-dependent manner. The increase in the D-amphetamine-induced overflow of dopamine seen in the substantia nigra and the striatum of the GDNF-treated animals could indicate that there may be an upregulation in the function and/or trafficking of dopamine transporters with the chronic infusion of GDNF. The increase in the potassium-evoked release of dopamine also suggests an upregulation in calcium-mediated dopamine release processes in the basal ganglia. As measured by in vivo microdialysis, GDNF was shown previously to augment basal and evoked overflow of striatal dopamine in aged rats $>3$ weeks after single intranigral injections (Hebert and Gerhardt, 1997), presumably long after clearance from the basal ganglia (Lapchak et al., 1997a). Thus, changes observed in basal and evoked dopamine release were conceivably maintained during washout in our aged animals. Clearly, additional studies are needed in aged nonhuman primates to investigate whether the increased overflow of dopamine seen in the basal ganglia during GDNF infusion is maintained in the absence of GDNF.

Similarly to the bilateral increase in evoked dopamine overflow in the substantia nigra, basal levels of extracellular dopamine were also significantly increased (more than twofold) in both the right and left substantia nigra of the GDNF-treated animals. No differences were observed between sides. These bilateral effects 
support the widespread distribution of the intraventricular delivery of GDNF and are consistent with the diffusion and/or retrograde transport of GDNF from the lateral ventricle or the striatum (adjacent to the ventricle) to the substantia nigra in both rats and monkeys (Tomac et al., 1995; Lapchak et al., 1997a, 1998). The bilateral effects observed in the substantia nigra could explain the bilateral improvement in motor functions, because there were no significant differences between the left- and rightlimb motor time values. Together, these observations support the view that GDNF can have bilateral effects when infused into one hemisphere of an animal (Gash et al., 1996; Grondin et al., 2002a). Changes in basal levels of extracellular dopamine in the substantia nigra may reflect changes in the synthesis of dopamine and/or storage of dopamine for somatodendritic release processes (Gerhardt et al., 2002). We and others have shown consistently that GDNF increases the number of TH-IR neurons in the substantia nigra of aged and MPTP-lesioned rhesus monkeys (Gash et al., 1996; Kordower et al., 2000; Ai et al., 2002; Grondin et al., 2002a). Thus, another possibility is that GDNF might have increased the number of TH-IR nigral neurons, leading to an increase in the basal and evoked release of dopamine in the basal ganglia. In our hands, the intrastriatal delivery of GDNF in aged monkeys resulted in an $18 \%$ increase in the number of TH-IR nigral neurons (Ai et al., 2002). Whether these effects of GDNF on the number of TH-IR nigral neurons can solely account for the twofold (up to 163\%) increase in dopaminergic functions (release) remains unclear. Alternatively, or in addition to its effects on the number of TH-IR nigral neurons, GDNF might be acting on midbrain dopaminergic neurons by potentiating neuronal excitability through a mechanism that involves activation of mitogen-associated protein kinase, leading to an increase in dopamine release (Yang et al., 2001). Clearly, the regulation of extracellular dopamine is complex, and additional research is needed to clarify the mechanisms through which GDNF is mediating its trophic effects in the host brain.

Dopaminergic projections from the substantia nigra pars compacta to the striatum (caudate nucleus and putamen) are well documented (Parent and Hazrati, 1995). Although the striatum is the major target of midbrain dopaminergic neurons, the globus pallidus (internal and external segments), the subthalamic nucleus, and the substantia nigra pars reticulata also receive significant dopaminergic input from the substantia nigra pars compacta (Smith and Kieval, 2000). Whether GDNF upregulates dopaminergic functions (release) in these nonstriatal areas, particularly in the globus pallidus, is an interesting question. Because these dopaminergic pathways play a major role in regulating motor movement (Obeso et al., 2000), we suggest that the effects of GDNF on the basal and stimulus-evoked release of dopamine in the substantia nigra and striatum may be responsible for the improvements in motor functions.

In summary, this study shows: (1) that GDNF increases the basal release of dopamine in the substantia nigra and the evoked release of dopamine in the striatum and substantia nigra of aged monkeys, (2) that GDNF can significantly improve motor functions in aged rhesus monkeys to a performance level comparable with that of young adults, and (3) that continuous stimulation of GDNF receptors may not be required for GDNF to improve motor functions effectively. The data also indicate that GDNF increases motor speed by increasing dopamine function in the aging nonhuman primate brain and support the hypothesis that functional changes in dopamine release in the basal ganglia may contribute to the motor dysfunctions characterizing normal aging in monkeys and possibly in humans.

\section{References}

Ai Y, Zhang Z, Grondin R, Gerhardt GA, Gash DM (2002) Effects of chronic intraputamenal delivery of GDNF on the nigrostriatal dopamine system in aging rhesus monkeys. Soc Neurosci Abstr 28:691.4.

Andersen AH, Zhang Z, Zhang M, Gash DM, Avison MJ (1999) Ageassociated changes in rhesus CNS composition identified by MRI. Brain Res 829:90-98.

Bachevalier J, Landis LS, Walker WC, Brickson M, Mishkin M, Price DL, Cork LC (1991) Aged monkeys exhibit behavioral deficits indicative of widespread cerebral dysfunction. Neurobiol Aging 12:99-111.

Bannon MJ, Poosch MS, Xia Y, Goebel DJ, Cassin B, Kapatos G (1992) Dopamine transporter mRNA content in the human substantia nigra decreases precipitously with age. Proc Natl Acad Sci USA 89:7095-7099.

Bennett DA, Beckett LA, Murray AM, Shannon KM, Goetz CG, Pilgrim DM, Evans DA (1996) Prevalence of parkinsonian signs and associated mortality in a community population of older people. N Engl J Med 334:71-76.

Bowenkamp KE, Lapchak PA, Hoffer BJ, Bickford PC (1996) Glial cell linederived neurotrophic factor reverses motor impairment in 16-17 month old rats. Neurosci Lett 211:81-84.

Brundin P (2002) GDNF treatment in Parkinson's disease: time for controlled clinical trials? Brain 125:1449-1451.

Cass WA (1996) GDNF selectively protects dopamine neurons over serotonin neurons against the neurotoxic effects of methamphetamine. J Neurosci 16:8132-8139.

Cass WA, Manning MW (1999) Recovery of presynaptic dopaminergic functions in rats treated with neurotoxic doses of methamphetamine. J Neurosci 19:7653-7660.

Emborg ME, Ma SY, Mufson EJ, Levey AI, Taylor MD, Brown WD, Holden JE, Kordower JH (1998) Age-related declines in nigral neuronal function correlate with motor impairments in rhesus monkeys. J Comp Neurol 401:253-265.

Fearnley JM, Lees AJ (1991) Aging and Parkinson's disease: substantia nigra regional selectivity. Brain 114:2283-2301.

Gash DM, Zhang Z, Ovadia A, Cass WA, Yi A, Simmerman L, Russell D, Martin D, Lapchak PA, Collins F, Hoffer BJ, Gerhardt GA (1996) Functional recovery in parkinsonian monkeys treated with GDNF. Nature 380:252-255.

Gash DM, Zhang Z, Gerhardt GA (1998) Neuroprotective and neurorestorative properties of GDNF. Ann Neurol 44:S121-S125.

Gash DM, Zhang Z, Umberger G, Mahood K, Smith M, Smith C, Gerhardt GA (1999) An automated movement assessment panel for upper limb motor functions in rhesus monkeys and humans. J Neurosci Methods 89:111-117.

Gerhardt GA, Cass WA, Henson M, Zhang Z, Ovadia A, Hoffer BJ, Gash DM (1995) Age-related changes in potassium-evoked overflow of dopamine in the striatum of the rhesus monkeys. Neurobiol Aging 16:939-946.

Gerhardt GA, Cass WA, Yi A, Zhang Z, Gash DM (2002) Changes in somatodendritic but not terminal dopamine regulation in aged rhesus monkeys. J Neurochem 80:168-177.

Glick SD, Dong N, Keller Jr RW, Carlson JN (1994) Estimating extracellular concentrations of dopamine and 3,4-dihydroxyphenylacetic acid in nucleus accumbens and striatum using microdialysis: relationships between in vitro and in vivo recoveries. J Neurochem 62:2017-2021.

Grondin R, Zhang Z, Beck K, Hilt D, Elsberry D, Gash DM (1998) Chronic intracerebral infusion of glial cell line-derived neurotrophic factor (GDNF) in parkinsonian rhesus monkeys. Soc Neurosci Abstr 24:42.

Grondin R, Zhang Z, Gerhardt GA, Gash DM (2000) Dopaminergic therapy improves upper limb motor performance in aged rhesus monkeys. Ann Neurol 48:250-253.

Grondin R, Zhang Z, Elsberry D, Gerhardt GA, Gash DM (2001) Chronic intracerebral delivery of trophic factors via a programmable pump as a treatment for parkinsonism. In: Parkinson's disease: methods and protocols, Vol 62 (Mouradian MM, ed), pp 257-267. Totowa, NJ: Humana.

Grondin R, Zhang Z, Cass WA, Ai Y, Maswood N, Andersen AH, Elsberry DD, Klein MC, Gerhardt GA, Gash DM (2002a) Chronic, controlled GDNF infusion promotes structural and functional recovery in advanced parkinsonian monkeys. Brain 125:2191-2201.

Grondin R, Zhang Z, Ai Y, Surgener SP, Loveland AD, Gerhardt GA, Gash DM (2002b) Chronic, pulsatile delivery of GDNF into the substantia nigra increases motor speed and dopamine levels in the basal ganglia of MPTP-treated parkinsonian monkeys. Soc Neurosci Abstr 28:691.3. 
Hebert MA, Gerhardt GA (1997) Behavioral and neurochemical effects of intranigral administration of glial cell line-derived neurotrophic factor on aged Fischer 344 rats. J Pharmacol Exp Ther 282:760-768.

Irwin I, DeLanney LE, McNeil T, Chan P, Forno LS, Murphy Jr GM, Di Monte DA, Sandy MS, Langston JW (1994) Aging and the nigrostriatal dopamine system: a non-human primate study. Neurodegeneration 3:251-265.

Kish SJ, Shannak K, Rajput A, Deck JHM, Hornykiewicz O (1992) Aging produces a specific pattern of striatal dopamine loss: implications for the etiology of Parkinson's disease. J Neurol Sci 53:347-357.

Kordower JH, Emborg ME, Bloch J, Ma SY, Chu Y, Leventhal L, McBride J, Chen E-Y, Palfi S, Zion Roitberg B, Brown WD, Holden JE, Pyzalski R, Taylor MD, Carvey P, Ling Z, Trono D, Hantraye P, Deglon N, Aebischer P (2000) Neurodegeneration prevented by lentiviral vector delivery of GDNF in primate models of Parkinson's disease. Science 290:767-773.

Kubis N, Faucheux MA, Ransmayr G, Damier P, Duyckaerts C, Henin D, Forette B, Le Charpentier Y, Hauw J-J, Agid Y, Hirsh EC (2000) Preservation of midbrain catecholaminergic neurons in very old human subjects. Brain 123:366-373.

Lapchak PA, Jiao S, Collins F, Miller PJ (1997a) Glial cell line-derived neurotrophic factor: distribution and pharmacology in the rat following a bolus intraventricular injection. Brain Res 747:92-102.

Lapchak PA, Miller PJ, Jiao S (1997b) Glial cell line-derived neurotrophic factor induces the dopaminergic and cholinergic phenotype and increases locomotor activity in aged Fischer 344 rats. Neuroscience 77:745-752.

Lapchak PA, Araujo DM, Hilt DC, Jiao S, Collin F, Miyoshi Y, Zhang Z, Gash DM (1998) Topographical distribution of $\left[{ }^{125} \mathrm{I}\right]$-glial cell line- derived neurotrophic factor in unlesioned and MPTP-lesioned rhesus monkey brain following a bolus intraventricular injection. Brain Res 789:9-22.

Obeso JA, Rodriguez-Oroz MC, Rodriguez M, Lanciego JL, Artieda J, Gonzalo N, Olanow CW (2000) Pathophysiology of the basal ganglia in Parkinson's disease. Trends Neurosci [Suppl] 23:S8-S9.

Ovadia A, Zhang Z, Gash DM (1995) Increased susceptibility to MPTP toxicity in middle-aged rhesus monkeys. Neurobiol Aging 16:931-937.

Parent A, Hazrati LN (1995) Functional anatomy of the basal ganglia. I. The cortico-basal ganglia-thalamo-cortical loop. Brain Res Brain Res Rev 20:91-127.

Smith C, Umberger G, Manning EL, Slevin JT, Wekstein DR, Markesbery WR, Zhang Z, Gerhardt GA, Gash DM (1999) A critical decline in fine motor hand movements in human aging. Neurology 53:1458-1461.

Smith Y, Kieval JZ (2000) Anatomy of the dopamine system in the basal ganglia. Trends Neurosci [Suppl] 23:S28-S33.

Tomac A, Widenfalk, Lin LF, Kohno T, Ebendal T, Hoffer BJ, Olson L (1995) Retrograde axonal transport of GDNF in the adult nigrostriatal system suggests a trophic role in the adult. Proc Natl Acad Sci USA 92:8274-8278.

Yang F, Feng L, Zheng F, Johnson SW, Du J, Shen L, Wu C, Lu B (2001) GDNF acutely modulates excitability and A-type $\mathrm{K}^{+}$channels in midbrain dopaminergic neurons. Nat Neurosci 4:1071-1078.

Zhang Z, Andersen A, Smith C, Grondin R, Gerhardt GA, Gash DM (2000) Motor slowing and parkinsonian signs in aging rhesus monkeys mirror human aging. J Gerontol Biol Sci Med Sci 55A:473-480. 